'key workers'. There is scarce evidence about the effect that the pandemic had on this group of workers as compared with people who were home working, furloughed or retired.

Objectives To compare measures of health and lifestyle amongst older key workers as opposed to other older adults working or not working during the pandemic.

Methods In February 2021 participants in the Health and Employment After Fifty (HEAF) study (aged 55-73) were sent an online survey, enquiring about changes to their mental/ physical health and lifestyle during the first UK lockdown (starting late March 2020). Logistic regression was used to explore the association between being a keyworker (healthcare/not healthcare) and adverse outcomes, with adjustment for age and sex. Participants in work but not identifying themselves as key workers were used as the reference category.

Results A total of 2,040 (46\%) returned a usable questionnaire and completed the question about key worker status. 281 were in work but not as key workers; 50 were key workers in healthcare; 298 were key workers not in healthcare; 1,411 were retired. Key workers were predominantly women. Key workers not in healthcare were more likely to see a worsening of mental health $(\mathrm{OR}=1.6 ; 95 \% \mathrm{CI} 1.0$ to 2.4$)$ physical health $(\mathrm{OR}=1.5 ; 95 \% \mathrm{CI} 1.0$ to 2.3$)$, to report that their diet was less healthy $(\mathrm{OR}=1.8 ; 95 \% \mathrm{CI} 1.2$ to 2.8$)$ and to report eating more than pre-lockdown $(\mathrm{OR}=1.6 ; 95 \% \mathrm{CI} 1.1$ to 2.4$)$ compared with participants in work but not as key workers. Associations were similar among key workers in healthcare, however they did not reach statistical significance.

Conclusion Being a key worker during the pandemic (especially not in healthcare) was associated with a deterioration of health and lifestyle choices.

\section{0-222 RISK OF INPATIENT CARE WITH COVID-19 IN DIFFERENT OCCUPATIONAL GROUPS: THE ROLE OF CLOSE HUMAN CONTACTS IN THE WORK ENVIRONMENT}

Maria Albin, Ahlbom Anders, Lars Alfredsson, Jenny Selander, Jonathan Lyström, ${ }^{1}$ Magnus Alderling. ${ }^{1}$ Karolinska Institutet, Sweden

\subsection{6/OEM-2021-EPI.29}

Introduction Several cross-sectional studies indicate that occupations such as physicians, nurses, dentists, physiotherapists, bus- and taxi drivers, bartenders, waiters, chefs and shop assistants either have higher risks of dying or be tested positive due to COVID-19.

Objectives The aim of this study was to analyse if employees in occupations characterized by working close to other people and/or being more exposed to diseases or infections are treated for inpatient care due to COVID-19 more often than other employees.

Methods Survey data regarding proximity to other people and to what extent individuals are exposed to diseases and infections were retreived from https://onet.rti.org/about.cfm. Analyses werebased on a prospective cohort comprising 1037811 residentsin Region Stockholm March 1, 2020, 18-64 years old and having a valid occupational code. The follow-up period was nine months. Quartiles were created, based on averages for each occupation regarding these questions. Adjusting factors were age, country of birth, daily smoking, overcrowding, household size and percentages in demographic areas being test positive to COVID-19. We calculated both IRR's and SMR's.

Results During the period, 2984 individuals received inpatient care for COVID-19. Higher risks were observed in the highest exposed quartiles after adjusting for confounders. These associations were found among both sexes, in all age groups and in each 3-month calender period with IRR's ranging from 1.68 to 2.58 compared to the least exposed quartile. SMR's calculated for each occupation revealed higher risks among e.g. physicians, nurses, assistant nurses, care assistants and traffic instructors with SMR's ranging from 138 to 272 .

Conclusions A strength of this study is the use of inpatient care as an outcome, and adjustment for individual confounders. The observation that the risk from close occupational contacts was not reduced over the observation period highlights the importance of further improvement of risk assessment and protective measures.

\section{0-332 AN UMBRELLA REVIEW OF THE WORK AND HEALTH IMPACTS OF WORKING IN A PANDEMIC ENVIRONMENT}

'Jonathan Fan, Sonja Senthanar, Robert Macpherson, Kimberly Sharpe, Cheryl Peters, Mieke Koehoorn, Chris McLeod. 'University of British Columbia, Canada

\subsection{6/OEM-2021-EPI.30}

Introduction The effects of the coronavirus disease 2019 (COVID-19) pandemic on work, employment and health are considerable. There is a need for actionable and targeted evidence that policy-makers, employers, workers and other stakeholders can use to ensure that work is safe and healthy not only during the COVID-19 pandemic, but also in its aftermath.

Objectives The purpose of this umbrella review is to inform evidence-based decision making and best practices for the work and health of workers during an epidemic/pandemic; and to identify research gaps to inform evidence needs for future studies and research funding priorities. We examined the evidence on the work and health impacts of working in an epidemic/pandemic environment; factors associated with these impacts; and possible risk mitigation or intervention strategies that address these factors or outcomes.

Methods We examined review articles published in MEDLINE, PsycINFO and Embase between 2000 and 2020. Data were extracted and analyzed using a narrative synthesis.

Results The search yielded 1,524 unique citations, of which 31 were included. The search yielded a large volume of reviews on mental health and infection risk to health care workers. Reviews identified a variety of individual, social, organizational and risk mitigation factors that influenced study outcomes. Equity considerations were only tangentially referenced in the included studies. Only a few reviews examined intervention strategies in the workplace, and none included long-term outcomes of exposure or work during an epidemic/ pandemic.

Conclusion Findings suggest a number of critical research and evidence gaps, including the need for reviews on occupational groups potentially exposed to or impacted by the negative work and health effects of COVID-19 in addition to health care workers, the long-term consequences of transitioning to the post-COVID-19 economy on work and health, and research with an equity or social determinants of health lens. 